Journal of Language \& Translation 11-2

September 2010, 179-205

\title{
The Distributivity of Collections Terms in Korean*
}

\author{
Eun-joo Kwak \\ Sejong University
}

\begin{abstract}
The plural morpheme -tul in Korean has been discussed to have two readings: a pluralizer and a distributor. A less discussed usage of $t u l$ is that it may be attached to a collection term to derive the member reading of a collection. However, not all terms that are considered as collective nouns are subject to individualization by $-t u l$. Thus, it must be discussed which nouns are individualized and what properties they share. This study presents four tests to define authentic collection terms and proposes that $-t u l$ has an additional reading of member specification function.
\end{abstract}

Keywords: distributivity, plural, collection, sum, group, member specification

\footnotetext{
* This work was supported by the Korea Research Foundation Grant funded by the Korean Government (KRF-2009-327-A00645).
} 


\section{Two Different Readings for Plural Collection Terms in Korean}

Although the interpretations of plural expressions have been discussed in diverse aspects, a great deal of focus lies on the semantics of count and mass nouns and their distributive readings. Interestingly, plurality and distributivity are not limited to non-collective count and mass nouns but are also applied to collection terms. Plural readings are delivered by plural forms with $-t u l$ or number-neutral forms without -tul. Just like non-collective NPs, collection terms may also occur with $-t u l$.
(1) Kacok-tul-i moyessta. family-P1-Nom gathered
'(The) families/family members gathered.'

When the collection term kacok is followed by -tul, it has an ambiguity between the plurality of collections and that of collection members. Kacok-tul may refer to either families or family members. Referring to plural collections, kacok-tul behaves like non-collective count nouns. However, in the reading of plural members of a collection, kacok-tul is distinguished from non-collective plural NPs. The collection kacok should be mapped to its members.

More interestingly, not all terms which seem to have plural members incur distributive readings in their plural forms. For example, kwukhoy 'the National Assembly' is a term that seemingly consists of plural members. However, its plural form kwukhoy-tul in (2) is not acceptable.

(2) \#Kwukhoy-tul-i moyessta.

National Assembly-P1-Nom gathered

'The members of the National Assembly gathered.' 
Although kwukhoy-tul may refer to plural entities of National Assembly in different countries, a single country cannot have more than one assembly. Thus, the reading of plural assemblies is not relevant in (2). The second possible reading regards the plural members of the National Assembly. However, kwukhoy-tul does not refer to the plural members of the collection, which is sharply contrasted with the member reading of kacok-tul in (1).

To account for the contrasting patterns of interpretations in (1) and (2), this study discusses the properties of collection terms in Korean and the semantics of the plural morpheme -tul. It is also discussed how plural-formed collections induce distributive readings based on the general theory of distributivity.

\section{The Distributivity of Collection Terms}

\subsection{Different Entities in an Interpretation Domain}

Distributivity is the application of a predicate to each individual or part of entities denoted by its argument. How distributivity holds in a sentence is related to what entities are involved in the sentence interpretation. This is further related to how an interpretation domain is structured. By the definition of distributivity, a single entity cannot induce distributivity. Thus, an interpretation domain should include not only singular entities but also plural ones. Since distributive readings may occur with both count and mass terms, the domain should include not only individuals for count terms but also materials for mass terms.

Link (1983) introduces a 'complete join semi-lattice' for an interpretation domain, based on a close similarity between plural and mass terms. ${ }^{1}$ A join semi-lattice structure is defined by a join opera-

\footnotetext{
${ }^{1}$ Quine (1960) argues for a cumulative reference property for plural and mass terms. If the property of being horses applies to the animals of one camp and to those of
} 
tion ' + ' and an individual part-of relation ' $\leq_{\mathrm{i}}$.' In this structure, the plural term John and Mary refers to a sum individual $\mathrm{j}+\mathrm{m}$, a larger entity that is derived from the joining operation of John's reference $j$ and Mary's reference m. An individual part-of relation exists between this newly generated sum $\mathrm{j}+\mathrm{m}$ and its atomic parts, $\mathrm{j} \leq_{\mathrm{i}} \mathrm{j}+\mathrm{m}$ and $m \leq_{i} j+m$. Here is a join semi-lattice for three individuals $j, m$, and $b$.

(3) a. Lattice for Individuals
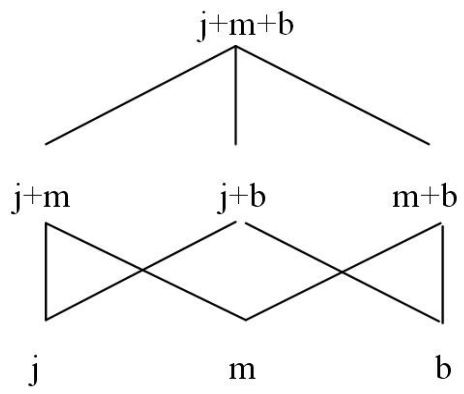

b. Lattice for Materials

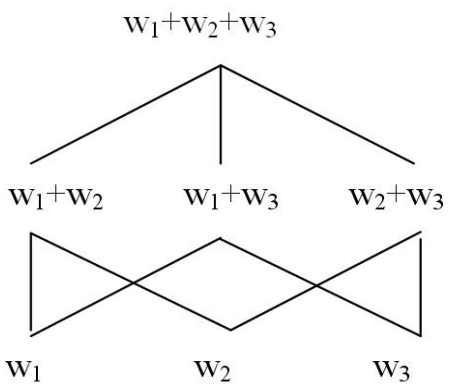

The first tier in (3a) consists of three atomic individuals $\mathrm{j}, \mathrm{m}$, and $\mathrm{b}$. Any two of these individuals may be joined together to make a sum individual such as $\mathrm{j}+\mathrm{m}, \mathrm{j}+\mathrm{b}$, and $\mathrm{m}+\mathrm{b}$. All three individuals are joined to make the maximal sum $\mathrm{j}+\mathrm{m}+\mathrm{b}$ in the third tier. Along with the lattice structure for plurals, Link also postulated another lattice structure for materials, which is defined by the join operation and a material part-of relation ' $\leq_{\mathrm{m}}$.' A cup of water $\mathrm{w}_{1}$ and another cup of water $\mathrm{w}_{2}$ may be poured into a basket to make a join material $\mathrm{w}_{1}+\mathrm{w}_{2}$. As with plurals, a material part-of relation holds between the sum

another camp, the property also applies to the animals in the two camps. In other words, the property of being horses applies to the cumulated reference of the animals in the two camps. As with plural terms, mass terms also show the cumulative reference property. If the property of being water applies to material $\mathrm{m}_{1}$ and also to material $\mathrm{m}_{2}$, the property applies to the cumulated reference of the two materials. 
material $\mathrm{w}_{1}+\mathrm{w}_{2}$ and its parts $\mathrm{w}_{1}$ and $\mathrm{w}_{2}, \mathrm{w}_{1} \leq_{\mathrm{m}} \mathrm{w}_{1}+\mathrm{w}_{2}$ and $\mathrm{w}_{2} \leq_{\mathrm{m}}$ $\mathrm{w}_{1}+\mathrm{w}_{2}$. A join semi-lattice for three materials $\mathrm{w}_{1}, \mathrm{w}_{2}$, and $\mathrm{w}_{3}$ is represented in $(3 \mathrm{~b})$, which has the same structure as the lattice for individuals.

A lattice structure with individuals and materials serves as a proper domain for the interpretations of plural and mass terms. However, this domain is not rich enough to deal with the interpretations of collection terms. Unlike non-collective singular or plural nouns, a collection term like a committee has an ambivalent property. Even a singular-formed collection term is not entirely singular but internally plural. Barker (1992) argues that a collection term may not be predicated by a singular NP.

(4) a. the group of armchairs/*armchair

b. one committee of women/*woman

c. an army of children $/{ }^{*}$ child

The group may take the plural member armchairs but not a singular one armchair. Likewise, a committee or an army may be predicated by the plural women or children but not singular. This shows that a collection term is semantically plural even in its singular form. Despite its internal plurality, a collection term may be pluralized as in committees to refer to plural collections. Since only singular count nouns may be pluralized, committee itself should be singular. Hence, a collection term is internally plural and externally singular in its semantics.

To reflect the dual nature of collection terms, the lattice structure for individuals needs to be expanded. Link (1984) and Landman (1989) assume two different categories of atoms, namely 'pure' and 'impure' atoms. Ordinary singular NPs refer to pure atoms, which do not have internal structures. However, collection terms refer to impure atoms, which are atomic but have an internal structure consisting of plural members. To implement a membership for a collec- 
tion term, Link (1984) introduces a group formation function ' $\uparrow$,' which maps a sum to a group, and Landman (1989) expands the structure with a member specification function ' $\downarrow$,' mapping a group to a sum of its members.

Let us assume that a police force consists of three officers $\mathrm{j}, \mathrm{m}$, and $b$ and that the same group of people also takes a class. Then, the three individuals $\mathrm{j}, \mathrm{m}$, and $\mathrm{b}$, and the groups of the police force and the class are assumed to be atoms in the domain represented in (5).

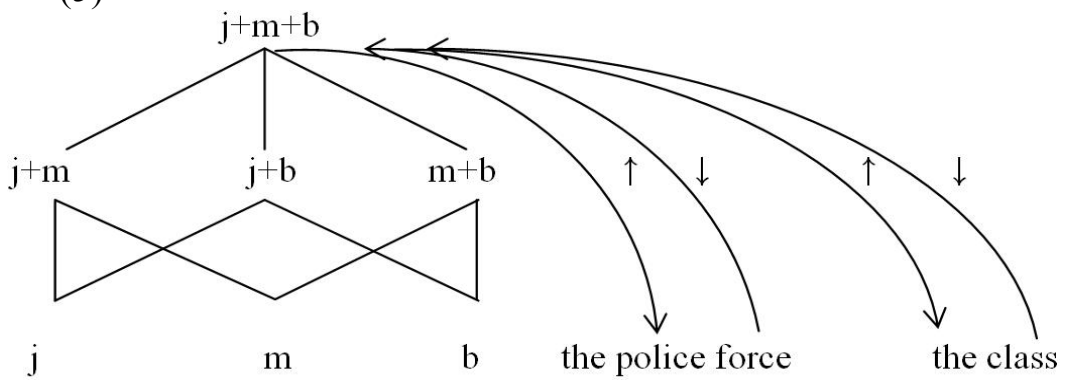

The sum $j+m+b$ is mapped to the group the police force or the class by $\uparrow .^{2}$ Likewise, the groups of the police force and the class are mapped to their members $\mathrm{j}+\mathrm{m}+\mathrm{b}$ by $\downarrow$. In this new structure, a collection term like the police force denotes an atom like an ordinary NP such as John. However, it has a plural internal structure with its members, which is specified by $\downarrow$. The dual nature of a collection term is successfully represented in this structure.

\footnotetext{
${ }^{2}$ A sum of individuals $\mathrm{j}+\mathrm{m}+\mathrm{b}$ is mapped to two groups the police force and the class in (5), which is against the notion of function. Landman (1989) argues that the problem of mapping one to many is due to the intensional property of group. The same sum of individuals may act as different groups depending on a given situation. If we reinterprete the extensional structure of (5) in the intensional setting, this mapping problem does not occur.
} 


\subsection{The Distributivity of Collection Terms}

The introduction of plurality in semantics involves distributivity. Depending on the lexical property of a predicate, a single individual may satisfy the action or state of the predicate. For instance, smiling applies only to a single individual. Hence, (6a) is construed to mean that the individual denoted by the student is part of the smiling set in the first order semantics.

(6) a. The student smiled.

b. The students smiled.

When the student is pluralized as in (6b), its denotation cannot be part of the smiling set. Since smiling cannot apply to plural entities as a whole, the smiling property needs to be distributed to each of the students. Hence, an implicit distributivity operator 'D operator' is introduced for the predicate as in (7a), which results in an interpretation in (7b).

(7) a. ${ }^{\text {D }}$ smiled'(the_students')

b. $\forall \mathrm{x}\left[\mathrm{x} \leq_{\mathrm{i}}\right.$ the_students' $\rightarrow$ smiled' $\left.(\mathrm{x})\right]$

The $\mathrm{D}$ operator has a universal quantification effect such that for every $\mathrm{x}$ that is part of the students, $\mathrm{x}$ smiled. The universal force of the $\mathrm{D}$ operator ensures the application of the predicate to each of the students.

A pure atom for an expression like $a$ student and a group for the one like a committee are atomic in a lattice structure as discussed in section 2.1. Thus, when a collection term is pluralized, it is either collective or distributive in its interpretation. ${ }^{3}$ For example, one in-

\footnotetext{
${ }^{3}$ The ambiguity of ( $\left.8 \mathrm{a}\right)$ comes from the collective property of the predicate. When the predicate is replaced with a distributive one like accepted the proposal, the resulting sentence has only a distributive reading.
} 
terpretation of (8a) is collective such that all the entities in the denotation of the committee participate in one event of gathering as represented in the logical form in (8b). ${ }^{4}$

(8) a. The committees gathered.

b. gathered'(the committees')

c. 'gathered'(the_committees')

$$
\left.=\forall \mathrm{x}\left[\mathrm{x} \leq_{\mathrm{i}} \text { the }{ }_{\text {committees' }} \rightarrow \text { gathered'( } \mathrm{x}\right)\right]
$$

(8a) also has a distributive reading with the application of the D operator on the predicate as in $(8 \mathrm{c})$. The universal quantification of the D operator applies the predicate over a sum of committees. The resulting interpretation is that for every $\mathrm{x}$ that is an individual-part of the committees, $x$ gathered.

Despite their atomic nature, a pure atom and a group are distinct entities in that only a group is internally plural with plural members. This distinct property is related to the fact that a group cannot occur in all the argument positions where a pure atom occurs. For instance, pass the exam may predicate the students but not a collection term like the class as shown by the contrast in (9a) and (9b).

(9) a. The students passed the exam.

b. *The class passed the exam.

c. The whole class passed the exam.

Passing the exam may be true for individual students but not for the class. Interestingly, when the collection term occurs with whole as in (9c), it may be predicated by the distributive predicate. (cf. Moltmann 1997, 2005; Morzycki 2001) The distributivity of whole

\footnotetext{
${ }^{4}$ Depending on theories, an event argument is posited for the interpretation of a sentence. Since the postulation of event does not make any difference in the discussion of this study, an event is not represented in the logical forms in (8).
} 
induces an interpretation that the property of the passing the exam is asserted for each member in the group denotation of the class. Thus, (9c) has a construal similar to that of (9a) in the context that the group denotation of the class is derived from the students. This means that the group denotation of the class must be shifted to a sum of its members with the occurrence of whole.

To get the meaning shift produced by whole, Kwak (2008) proposes that whole be construed as the member specification function $\downarrow$, taking a group as an argument and mapping it to a sum of its members. Suppose that the class consists of three individuals, John, Mary, and Bill. Then, the whole class is interpreted as in (10).

(10) $[[$ the whole class $]]=\downarrow$ (the_class' $)=\mathrm{j}+\mathrm{m}+\mathrm{b}$

By taking the collection term the class as an argument, whole maps it to a sum of its members, $\mathrm{j}+\mathrm{m}+\mathrm{b}$. The members of the class $\mathrm{j}+\mathrm{m}+\mathrm{b}$ are the students in the class. Hence, the whole class has an interpretation similar to that of the students. Since the derived entity by whole is a sum, the distributive operator D is introduced in the sentence with a distributive predicate.

(11) [[the whole class passed the exam $]]$

$$
\begin{aligned}
& ={ }^{D} \text { passed_the exam' }(\downarrow(\text { the_class' })) \\
& =\forall \mathrm{x}\left[\mathrm{x} \leq_{\mathrm{i}} \downarrow \text { (the_class') } \rightarrow\right. \text { passed_the_exam'(x)] }
\end{aligned}
$$

With the universal quantification of the D operator, (11) is interpreted such that for every $\mathrm{x}$ that is an individual-part of the members of the class, $x$ passed the exam. A collection term is distinguished from a non-collective noun in that it has an internal structure with plural members. Thus, a collection term may induce a distributive reading even in its singular form by the application of member specification function, which may be lexicalized by whole in English. 


\section{The Distributivity of Collection Terms in Korean}

\subsection{The Plural Morpheme - Tul as a Pluralizer and a Dis- tributor}

Distributivity is closely related to the topic of plurality in that the distributive reading is available only when there is more than one entity in the discourse. Plurality is further connected to the countability of nouns since only count nouns may be pluralized. Countability and plurality are straightforward in languages where plural NPs are morphologically distinguished from singulars and mass nouns as in English. However, these topics are controversial issues in Korean, in which plural NPs do not always occur in plural forms. ${ }^{5}$

When haksayng 'student' is followed by the plural morpheme $t u l$, it cannot be used for a single student. ${ }^{6}$ It refers to a plural entity without ambiguity.
(12) a. haksayng-tul
'(the) students' student-P1

\footnotetext{
${ }^{5}$ The definiteness of a plural-formed NP is also controversial. Song (1994) argues for the definite interpretation of a plural-formed NP. However, Kwak (2003) and Jun (2004) argue that plural-formed NPs in Korean have ambiguous readings between definite and indefinite NPs. Since this is beyond the scope of this study, it is assumed that plural-formed NPs are ambiguous without further discussion.

${ }^{6}$ It has been generally assumed that nouns are divided into count and mass. However, even this general assumption is challenged depending on theories. According to the typological study of Chierchia (1998a,b), languages that allow nominals to occur in argument positions without a determiner have only mass nouns. Chinese and Japanese are classified as argumental languages with only mass nouns. Korean should also be an argumental language without count nouns because NPs without a determiner may occur in argument positions. Moving further from this typological study, Park (2008) proposes that nouns in Korean are all mass and that $-t u l$ is unambiguously used as a distributor. It is still an unresolved issue that argumental languages like Korean have only mass nouns. Hence, this possibility is not further pursued in this study.
} 

b. haksayng
'a/the student(s)' student

However, the interpretation of the bare-formed NP haksayng is not limited to a singular. It may be used to denote a plural entity of students. Plural-formed NPs like haksayng-tul are discussed to denote sums of individuals in much of the literature. (cf. Kang 1994; Im 2000; Baek 2002; Kwak 1996, 2003; Jun 2004; Noh 2008) However, the semantics of bare-formed NPs is too controversial to reach any consensus among semanticists. Some argue for the sum readings of bare-formed NPs while others propose group denotations for these NPs. Even plural interpretations for bare-formed NPs are denied depending on semanticists. (cf. Noh 2008) Since this study focuses on the semantics of plural-formed collection terms, the interpretations of bare-formed NPs are not further discussed. Along with the previous analyses, it is assumed that plural-formed NPs denote sums and that $-t u l$ functions as a pluralizer for sum readings.

In addition to a pluralizer, -tul is also used as a distributor. Unlike the plural morpheme $-S$ in English, -tul may be attached to mass terms and adverbials as well as count terms.

(13) a. Yeki ceki-se mwul-tul-i ssotacyessta. here there-Loc water-Pl-Nom be poured 'Water was poured here and there.'

b. Manhi-tul mekeyo.

much eat 'Help yourself.'

The mass term $m w u l$ 'water' in (13a) denotes a non-atomic entity of material, so it cannot be pluralized by $-t u l$. Since the adverbial manhi 'much' in (13b) predicates the predicate mekeyo 'eat,' manhitul itself does not denote a sum of atoms. It is generally accepted that $-t u l$, attached to the adverbial, originates from a plural-formed 
190 The Distributivity of Collections Terms in Korean

subject (or object). (cf. Song 1975, 1993, Lee 1982, Im 2000) Thus, -tul for the mass term and the adverbial does not function as a pluralizer to denote a sum of atoms. It is more likely to be used as a distributor. Mwul in (13a) is distributed over locations as expressed by yeki ceki-se 'here and there,' and manhi in (13b) is distributed over the deleted plural subject. Although the semantic function of tul in this usage is still controversial, one possible explanation is that -tul is the morphological realization of the D operator.

\subsection{Previous Analyses on the Distributivity of Collection Terms}

The function of $-t u l$ is divided into two: a pluralizer for count terms and a distributor for mass terms and adverbials. However, the occurrence of $-t u l$ is not confined to these categories. According to Im $(1979,2000)$, -tul may be attached to collection terms like kacok 'family.'

(14) Kacok-tul-i moyessta.

family-P1-Nom gathered

'(The) families/family members gathered.'

When the collection term kacok is followed by -tul, one interpretation is a plural entity of families. The function of $-t u l$ in this reading is a pluralizer just like the one for non-collective count terms. Unlike non-collective count terms, the plural-formed collection term has an additional reading of a plural entity of family members. Given the ambiguity of the collection term, (14) is construed as a gathering of multiple families or as a gathering of one family with plural family members. Im enumerates more examples of collection terms which have member readings with the occurrence of -tul: kwukmin 'a people,' kwuncwung 'a crowd,' and taycwung 'the public.'

There are basically two possible ways to derive the member 
reading of a plural-formed collection term. One is to postulate the ambiguity of the collection term. For example, the lexical meaning of kacok is assumed ambiguous between a collection of a family and an individual family member. On this assumption, the role of $-t u l$ may remain a pluralizer. It induces pluralized readings of collections or collection members. The other way is to assume the ambiguity of $-t u l$. $-T u l$ has an additional function other than a pluralizer while the collection term kacok is unambiguously construed as a collection. Im (2000) does not assume explicitly that kacok is lexically ambiguous. He proposes that -tul disintegrates a collection and individualizes its members. This process is a kind of pluralization because the plural-formed collection term refers to a set of individualized members. Hence, Im is more likely to focus on the uniform interpretation of $-t u l$ for non-collective count terms and collection terms.

Im (2000) further argues that not all collection terms are individualized by $-t u l$. A common property of collection terms is that they consist of plural members. According to this criterion, Im argues that hamtay 'fleet,' tanchey 'team,' and ciphap 'set' are all collection terms because their denotations are based on plural members. However, none of these terms induce member readings with the occurrence of $-t u l$.

(15) a. \#Hamtay-tul-i kotongsoli-lul naynta. fleet-Pl-Nom whistle-Acc blow

b. *Tanchey-tul-i sangnyanghata. organization-Pl-Nom kind

c. *Ciphap-tul-i ccakswu-ita. set-Pl-Nom even number-be

Hamtay-tul may denote a plural entity of fleets, but it is not construed as a plural entity of ships in a fleet. Thus, (15a) does not have a reading that the ships of a fleet blow a whistle. Similarly, tancheytul is not construed as the members of an organization, so sangn- 
yanghata 'kind,' a property of individuals, is not an appropriate predicate for it. Although ciphap may consist of plural elements, ciphap-tul is not interpreted as a plural entity of the elements in a set. The awkwardness in (15a)-(15c) contrasts with the ambiguity in (14).

To account for the different interpretations of plural-formed collection terms, Im (2000) argues that a member reading is limited to a certain group of collection terms which satisfies a restriction like (16).

(16) Restriction on the Individualization of a Collection Term A collection term is individualized by -tul only when its member has the same name as the collection or when individuality is part of the property of a collection.

According to this restriction, kacok 'family' consists of kacok 'family members,' so it can be individualized by -tul. However, hamtay 'fleet' is not used to denote a member in a fleet, and tanchey 'team' is limited to the denotation of a collection. These terms do not meet the restriction in (16) and cannot refer to their members with the occurrence of -tul. It this is true, it is questionable why individualization should be restricted by the ambiguity of a collection term. If this is not an ad-hoc restriction, the function of $-t u l$ is a pluralizer just as for non-collective count terms. Another problem with (16) is that it does not account for the individualization of collection terms like kwuncwung 'a crowd' and taycwung 'the public.' Im admits that kwuncwung and taycwung cannot be used to denote their members. However, he argues that these terms denote just a gathering of people without having additional properties by themselves, which is why they can be individualized by -tul. This account is neither persuasive nor subsumed under the restriction in (16).

Kim (2005) provides a separate account for the individualization of a collection term. First, based on the co-occurrence of a quantifier, he argues for the member reading of a plural-formed collection term. 


(17) a. taytaswu-uy kwukmin-tul 'most of a people'
most-Acc people-P1
b. manhun kwukmin-tul 'many of a people'
many people-P1

When kwukmin-tul occurs with a quantifier like taytaswu 'most' or manhun 'many,' it is not construed as a quantification over collections such as most peoples or many peoples. The quantification of (17a) and (17b) is over the members in a people. This shows that $k w u k$ min-tul is construed as a plural entity of members and then quantified by the quantifiers.

Second, Kim divides collection terms into two categories: collections terms and group terms. Collection terms denote human collections that consist of human members. On the other hand, group terms denote non-human collections although the collections consist of human members. Kacok, kwukmin, kwuncwung, and taycwung are categorized as collection terms, while tanchey is a group term. Kim argues that member readings with the occurrence of $-t u l$ are limited to collection terms. Group terms are not individualized with -tul. Although this categorization makes it clearer what kind of collection terms do not have member readings, it is still problematic in two respects. One is why human and non-human collections should be distinguished for the individualization of -tul. If the humanness of collections is not proved to be a crucial feature for individualization, this account is not persuasive. Another is that collections with nonhuman members are not categorized in this analysis. Hamtay and ciphap are generated from non-human members, but they are still interpreted as collections with plural members. However, since they are not explicitly categorized in Kim (2005), it is not explained why they do not have member readings in plural forms.

Im (2000) and Kim (2005) make an interesting observation that plural-formed collection terms may have individualized member 
readings and that only a certain group of collection terms is subject to individualization by $-t u l$. However, they do not provide a satisfying criterion to define a group of individualized collection terms. Moreover, it requires more study to account for how -tul individualizes a collection and whether -tul has a separate reading in addition to a pluralizer and a distributor.

\subsection{Properties of Collection Terms in Korean}

Barker (1992) defines a collection term as a noun which can take an of phrase that contains a plural complement but not a singular complement. $^{7}$

(18) a. a committee of women/*woman

b. a picture of horses/horse

The collection term committee is modified by the plural complement of women but not by the singular of woman. However, the non-collective noun picture may be modified by either the plural of horses or the singular of horse. Since the denotation of a collection term is generated from plural members, its member specification by of phrase is limited to plural entities.

Given the definition of collection terms in English and the test to confirm their collective properties, a similar test may be adopted for collection terms in Korean. First, since the denotation of a collection term is based on its plural members, member specification should be available for a collection term. For example, when kacok 'family' consists of foreigners, its member specification may be done by modification as in (19a).

\footnotetext{
${ }^{7}$ Barker (1992) uses an expression of group terms instead of collection terms because these terms denote groups rather than sums. Group terms are not used to avoid confusion.
} 
(19) a. oykwukin kacok 'a family of foreigners' foreigner family

b. yeseng chwulyencin 'a cast of female actors' female cast

c. cangayin chengcwung 'an audience of handicapped people' handicapped people audience

Similarly, when a cast consists only of female actors, this relation may be specified by modification as in (19b). Member specification for chengcwung 'audience' is also possible as in (19c).

Note that not all nouns that have been regarded as collection terms allow member specification. Although tanchey 'organization' or wiwenhoy 'committee' may be modified by an expression like sopica 'consumer,' this is not a case for a collection and its members.

(20) a. sopica tanchey/wiwenhoy consumer organization/committee '\#an organization/committee of consumers'

b. yeseng hakhoy/*kwukhoy female academic society/national assembly '\#an academic society/national assembly of women'

The organization or committee in (20a) is understood as the one for consumers, in which sopica provides a purpose for the organization or committee rather than its members. Likewise, even though an academic society or the national assembly consists only of female members, this relation is not expressed as in (20b). Occurring with hakhoy 'academic society,' yeseng 'women' works as the academic field or purpose of the society. Since kwukhoy 'national assembly' cannot take yeseng as its purpose, yeseng kwukhoy itself sounds awkward. Group terms in Kim (2005) are defined as non-human collections with human members. In other words, group terms de- 
note social entities that have human members in the real world. Kim argues that group terms show different properties from collection terms without further explanation. The different modification and interpretation for terms of social entities shows that these terms do not have an internal structure with plural members. Unlike authentic collection terms that have plural members, terms of social entities are pseudo-collection terms that are not treated as collections linguistically.

In addition to terms of social entities, hamtay 'fleet' and pwutay 'army' are not treated as collection terms with members.

(21) a. hangkongmoham hamtay '\#a fleet of aircraft carriers' aircraft carrier fleet

b. nakhasan pwutay '\#an army of parachute' parachute army

When hamtay is modified by hangkongmoham 'aircraft carrier,' it does not mean that the fleet consists only of aircraft carriers. Rather, it denotes a sum of ships that are needed for the proper function of an aircraft carrier. Thus, the modifier hangkongmoham is more like a purpose for hamtay rather than its members. Similarly, the modifier nakhasan does not specify the members of an army in (21b).

Second, the internal structure of a collection term is also accessed by the specification of the number of collection members. For example, when a family has three members, this relation may be expressed as in (22a).

(22) a. sey myeng-uy kacok 'a family of three people' three Cl-Poss family

b. payk myeng-uy kwuncwung 'a crowd of a hundred people' hundred Cl-Poss crowd

c. sachenman kwukmin 'a people of forty million' forty million people 
Other collection terms such as kwuncwung 'crowd' and kwukmin 'people' may occur with expressions specifying the size of the collections as in (22b) and (22c). However, terms of social entities and ciphap 'set' may not be modified by expressions for the number of their members.

(23) a. \#sampayk myeng-uy kwukhoy/tanchey

300 Cl-Poss national assembly/ organization

'\#a national assembly/organization of 300 people'

b. \#yel kay-uy ciphap

10 Cl-Poss set

'\#a set of ten elements'

(23a) cannot be used even in a situation when the national assembly or organization consists of three hundred members. (23b) may be understood as ten sets but not as a set with ten elements. Thus, the size of its members may not be specified for kwukhoy, tanchey, and ciphap in the same way as the authentic collection terms in (22). If we assume that these terms are not collective, it is naturally explained why this category of nouns behaves in a different way.

Finally, an authentic collection term may be followed by a plural anaphora to refer to its plural members, but a pseudo-collection term may not.

(24) a. Kacok-i moyessta. Kutul-i cenyek-ul mekessta. family-Nom gathered they-Nom dinner-Acc had 'A/the family gathered. They had a dinner.'

b. Kwuncwung-i moyessta. Kutul-i soli-lul chyessta. crowd-Nom gathered they-Nom shout-Acc did 'A/the crowd gathered. They shouted.' 
198 The Distributivity of Collections Terms in Korean

$$
\begin{aligned}
& \text { (25) a. \#Tanchey-ka uykyen-ul nayssta. } \\
& \text { organization-Nom opinion-Acc made } \\
& \text { Kutul-un panday-lul hassta. } \\
& \text { they-Top opposition-Acc did } \\
& \text { '\#An/the organization made an opinion. They } \\
& \text { opposed (pro).' } \\
& \text { b. \#Pwutay-ka itong-ul hayssta. Kutul-i cichyessta. } \\
& \text { army-Nom move-Acc did they-Nom got tired } \\
& \text { '\#An/the army moved. They got tired.' }
\end{aligned}
$$

The authentic collection terms like kacok or kwuncwung may be followed by the plural anaphora kutul 'they,' which denotes the plural members of the family or the crowd as shown in (24). However, the pseudo-collective nouns tanchey and pwutay in (25) may not be followed by the plural anaphora. The acceptability differences in (24) and (25) provide more evidence for the internal plurality of the collection terms and non-collective properties of the pseudocollection terms.

As noted in section 3.1, the plurality of Korean is not determined only by the plural morpheme. Bare-formed NPs may be used to denote either singular or plural entities in Korean. Thus, a noncollective noun like haksayng may be incorrectly judged to be a collection term according to the above tests.

(26) a. oykwukin haksayng foreigner student 'foreign students'

b. sey myeng-uy haksayng three Cl-Poss student 'three students'

c. Haksayng-i moyessta. Kutul-i soli-lul chyessta. student-Nom gathered they-Nom shout-Acc did 'Students gathered. They shouted.' 
When a sum of students shares the property of foreigner, it may be expressed by modification as in (26a). How many entities are included in the denotation of haksayng may be specified as in (26b). Finally, the plural anaphora kutul may follow haksayng to denote a plural entity of students as in (26c). Apparently, a collection term like kacok and a non-collective one like haksayng do not show any difference linguistically, which casts a doubt on the plausibility of the tests as the common property of collection terms.

Although a non-collective noun has an ambiguity between a singular and plural denotation, it is disambiguated with the occurrence of the demonstrative determiner $k u$. (cf. Song 1975; Nemoto 2005)
(27) a. ku haksayng
'the student' the student
b. ku haksayng-tul
'the students' the student-PL

Although haksayng may denote either a single student or multiple students, the interpretation of ku haksayng is limited to a single student. Similarly, the plural one in (27b) denotes only a sum of students. Unlike the atomic interpretation of ku haksayng, the collection term $k u$ kacok stills denotes a collection with plural members.
(28) ku kacok
'the family'

$K u$ kacok does not refer to a single family member but a family with plural members. As Barker (1992) argues, an authentic collection term always takes plural members, but a non-collective noun does not. If haksayng were an authentic collection noun, ku haksayng should be able to denote a plural entity like the non-demonstrative haksayng.

In the previous analyses, it is not explicitly defined which nouns are authentic collection terms that may be individualized by the oc- 
currence of -tul. This study presents four tests to define a group of authentic collection terms that are distinguished from pseudocollective nouns and non-collective bare nouns. Following Barker (1992), the four tests are based on the internal plurality of collection terms.

\subsection{The Distributivity of Collection Terms}

The plural morpheme $-t u l$ is ambiguous between a pluralizer and a distributor as discussed in section 3.1. Given the individualization of collection terms by $-t u l$, it needs to be answered whether $-t u l$ has an additional interpretation. In the analysis of Im (2000), it is not clearly stated whether -tul has a separate reading for collection terms. Although he admits that $-t u l$ has a role of individualizing the members of a collection, Im focuses on the plurality of a resulting set by individualization.

Suppose that $-t u l$ has no additional interpretation other than a pluralizer and a distributor. Then, the individualizing function of $t u l$ must be attributed to one of the two. Since $-t u l$ as a distributor is restricted to grammatical categories other than nouns, -tul as a pluralizer is more suitable for the individualization of collection terms. As noted by Im (2000), the interpretation of kacok-tul in (29a) is ambiguous between families and family members.

(29) a. Kacok-tul-i moyessta.. 'A/the family gathered.' family-Pl-Nom gathered

b. Kwuncwung-tul-i moyessta. 'A/the crowd gathered.' crowd-Pl-Nom gathered

To derive the ambiguity of kacok-tul, two possible approaches are available. One is to assume the ambiguity of kacok between a collection and a member, while the other is to postulate an additional reading for $-t u l$. The ambiguity of a collection term seems to 
work for kacok but not for kwuncwung. Unlike kacok-tul, kwuncwungtul does not denote plural collections. It only has a plural member reading such as people in a/the crowd. Suppose that the member reading of a pluralized collection term is based on its lexical ambiguity. Then, it is not explained why kwuncwung-tul has a member reading despite a lack of lexical ambiguity.

Taking the second option, let us assume that -tul has an additional interpretation to derive the member reading of a collection term. In the interpretation domain described in section 2.1, a collection term denotes an impure atom of a group. For example, kacok may denote a group $f_{1}$. The member reading of a group is derived by the member specification function $\downarrow$ in this framework. Thus, it is proposed that $-t u l$ should be used as $\downarrow$ occurring with a collection term.

$$
\begin{aligned}
& \text { (30) a. }[[\text { tul }]]=\downarrow \\
& \text { b. }\left[[\text { kacok-tul }]=\downarrow \text { the family' }=\downarrow f_{1}=a+b+c\right. \\
& \text { c. }[[\text { kacok-tul-i moyessta }]]=\text { gathered' }(\downarrow \text { the family' }) \\
& =\text { gathered' }\left(\downarrow f_{1}\right)=\text { gathered' }(a+b+c) \Rightarrow{ }^{\text {Dathered' }}(a+b+c) \\
& \text { d. }\left[[\text { kacok-i moyessta] }]=\text { gathered'(the_family') }=\text { gathered'( } f_{1}\right)
\end{aligned}
$$

Under this proposal, the member reading of kacok-tul is derived by the application of $\downarrow$ as in (30b). $\downarrow$ maps a group $\mathrm{f}_{1}$ to a sum of its members, say $a+b+c$, in the situation that $f_{1}$ consists of three atomic individuals of $\mathrm{a}, \mathrm{b}$, and $\mathrm{c}$. When kacok-tul combines with a predicate like moyessta, the D operator is introduced due to the plural property of the members and induces a reading that each of the family members is involved in the event of the gathering. When -tul is not attached to kacok, there is no process of member specification. Thus, the sentence in (30d) is simply construed that a group is the agent of a gathering event. No further implication is involved in this group reading. Similar member specification may be applied to other collection terms like kwuncwung.

In the previous section, pseudo-collective nouns are distin- 
guished from authentic collection terms. For example, kwukhoy 'national assembly' is a pseudo-collective noun referring to a social entity. Since this is not a collection term, it has no internal structure with plural members. This means that when -tul applies to kwukhoy, it has no proper reading.

(31) a. $[[$ kwukhoy-tul $]]=\downarrow$ the_national_assembly $=$ ?

b. \#Kwukhoy-tul-i moyessta.

National Assembly-Pl-Nom gathered

'The members of the National Assembly gathered.'

Since kwukhoy is not a collection term, it does not denote a group. Its denotation must be a pure atom with no internal structure. The function $\downarrow$ cannot map it to any members, so kwukhoy-tul itself is meaningless and any sentence with this expression like (31b) sounds awkward.

As discussed in section 2.2, the members of a collection may be specified by whole in English. Although the collection the class cannot occur with a predicate for individuals like pass the exam, the whole class may co-occur with it. Hence, the whole class has an interpretation similar to the students in the situation that the denotation of the students constitutes the members of the class. Likewise, the member specifier -tul has a role similar to whole. It takes a collection and maps it to its members. This is why kacok-tul has a reading similar to sikkwu-tul 'family members.'

\section{Conclusion}

The plural morpheme -tul in Korean has been discussed to have two major functions: a pluralizer for count nouns and a distributor for other grammatical categories. Im $(1979,2000)$ has observed that collection terms in Korean may be followed by -tul to deliver their member readings and that not all collection terms are subject to in- 
dividualization by -tul. Kim (2005) provides further support for this observation and argues that group terms, which denote non-human collections generated from human members do not undergo individualization by $-t u l$. Although these analyses have made an interesting observation on the use of -tul, they neither make clear what kind of collection terms are individualized nor specify what interpretation $-t u l$ should be assigned for the individualization of collection terms.

Barker (1992) argues that collection terms are internally plural and always take a plural complement. Based on this definition, the present study provides common properties of collection terms that are individualized by $-t u l$. It is also argued that nouns that seem to have plural members in the real world may not be treated as collection terms linguistically. Group terms in Kim (2005) denote social entities that seem to have human members in the real world. Given the tests for collection terms, terms of social entities are not related with their members in linguistics, so they do not undergo individualization by $-t u l$. Other nouns that are not individualized are also filtered out by the proposed tests.

Given the new definition for collection terms, it is proposed that $-t u l$ has an additional reading of member specification function. The plural member reading of a collection term with -tul is the result of the application of the member specification function, which is independently required in an interpretation domain. Since this function may apply only to an authentic collection term, it does not apply to non-collective nouns. This is why pseudo-collective nouns are not individualized by $-t u l$.

\section{References}

Baek, M. 2002. Hankwuke Pokswu Uymi Yenkwu (A Study of Plural Meaning in Korean). Discourse and Cognition 9, 59-78. 
204 The Distributivity of Collections Terms in Korean

Barker, C. 1992. Group Terms in English: Representing Groups as Atoms. Journal of Semantics 9, 69-93.

Chierchia, G. 1998a. Plurality of Mass Nouns and the Notion of Semantic Parameter. In S. Rothstein (ed.), Events and Grammar. Kluwer, Dordrecht.

Chierchia, G. 1998b. Reference to Kinds across Languages. Natural Language and Semantics 6, 339-405.

Im, H. 1979. Pokswuseng-kwa Pokswuhwa (Plurality and Pluralization). Hankwukhaknonchong 2, 179-218.

Im, H. 2000. Pokswu Phyoci 'tul'kwa Sakenseng (The Plural Marker-tul and Eventuality). Aysanhakpo 24, 3-50.

Jun, Y. 2004. Hankwuke-uy Pokswuseng-kwa Chongchingseng / hancengseng (Plurality and Its Effects on Genericity and Definiteness in Korean). Language and Information 8, 27-44.

Kim, J. 2005. Pokswu Kwanlyen Hyengthay '-tul'ey Tayhaye (On the Plural Form of 'tul'). Wulimal Yengwu. Seoul: Thayhaksa, 85-107.

Kwak, E. 1996. The Event-dependency of Noun Phrases. Ph. D. thesis, Brown University.

Kang, B. 1994. Plurality and Other Semantic Aspects of Common Nouns in Korean. Journal of East Asian Linguistics 3, 1-24.

Kwak, E. 2003. Interpretations of Plural Nouns Phrases in Korean. Korean Journal of Linguistics (Enehak) 35, 3-36.

Kwak, E. 2008. Whole as a Semantic Pluralizer. Language and Information 12, 67-83.

Landman, F. 1989. Groups I \& II. Linguistics and Philosophy 12, 559-606 \& 723-744.

Lee, N. 1982. Tanswu-wa Pokswu (Singular and Plural). Kwukehak $11,117-141$.

Link, G. 1983. The Logical Analysis of Plurals and Mass Terms: A Lattice Theoretical Approach. In N. Bäuerle et al, eds., Meaning, Use, and Interpretation of Language. Walter de Gruyter, Berlin, 302-323. 
Link, G. 1984. Hydras on the Logic of Relative Construction with Multiple Heads. In F. Landman, et al. (eds.), Varieties of Formal Semantics. Dordrecht: Foris, 245-257.

Moltmann, F. 1997. Parts and Wholes in Semantics. Oxford: Oxford University Press. . 2005. Part Structures in Situations: The Semantics of Individualand Whole. Linguistics and Philosophy 28, 599-641.

Morzycki, M. 2001. Wholes and Their Covers. Proceedings of SALT 12, UC San Diego, Ithaca: Cornell University Press.

Nemoto, N. 2005. On Mass Denotations of Bare Nouns in Japanese and Korean. Linguistics 43, 383-413.

Noh, E. 2008. Hankwukeuy Mwuphyohengkwa 'Tul'-Pokswuhenguy Uymi (A Study of the Meaning of Korean ø-marked and 'tul-Marked Nouns). Discourse and Cognition 15, 43-62.

Park, S. 2008. Functional Categories: The Syntax of DP and DegP. $\mathrm{Ph}$. D. thesis, University of Southern California.

Quine, V. 1960. Word and Object. Cambridge: MIT Press.

Song, S. 1975. Rare Plural Marking and Ubiquitous Plural Marker in Korean. Language Research 11, 77-86.

Song, S. 1993. Hankwuke Mwunpepuy Say Comyeng (A New Perspective on Korean Grammar). Seoul: Cisiksanepsa.

Eun-joo Kwak

English Department Sejong University

98 Kunja-dong, Kwangjin-ku Seoul, Korea 143-747

Phone: 822-3408-3633; Email: ejkwak@sejong.ac.kr

Received Jul. 2010; Reviewed Aug. 2010; Revised version received Aug. 2010. 